\title{
Editorial
}

\section{Paediatric premedication}

In this issue of the Journal McMillan et al. ${ }^{1}$ describe the use of midazolam to premedicate children. They demonstrate that oral midazolam is safe and effective for children aged one to six years scheduled for ambulatory surgery. The study is well conducted and its message is comforting. Children can be sedated preoperatively with ease and safety. This article adds to the growing body of paediatric literature on the pharmacological preparation of the child before surgery. The practising anaesthetist now has an increasing number of agents and routes well described in the literature and should be able to determine appropriate treatment for the child.

The use of premedication in children has come full circle from the heavy sedation of the early years to the unpremedicated trend of the 70's and 80's and now a trend to preinduction sedation. Does the increased number of studies on paediatric sedation represent a real advance in the management of patients or is it merely a pharmacological exercise to determine which drugs can be absorbed through a variety of mucosal surfaces? On review of the indications for premedication we can appreciate that although they have changed for many of our patients they still exist.

Historically, premedication of children was a necessity for the safe conduct of the subsequent general anaesthetic. In a classic paper, Waters ${ }^{2}$ expressed the strong opinion that children should receive premedication like the adults of that period, that is, with morphine and scopolamine. At a time when most muscle relaxation was provided by volatile anaesthetics, morphine was used to decrease the depth of inhalational agent required and to produce improved cardiovascular stability. Scopolamine as a drying agent was extremely important in an era when ether produced copious secretions. In contrast to today, the child of Waters' practice was more often sick and the airway was usually unprotected. With the subsequent development of muscle relaxants and newer inhalational agents the absolute requirement for these premedicants gradually disappeared in the paediatric patient.

From the Department of Anaesthesia, Children's Hospital, Winnipeg, Manitoba.
There are indications, even today, for premedicants which improve the safety of general anaesthesia. Atropine is given to dry secretions in the difficult airway and to maintain heart rate during induction of anaesthesia in the infant. Ketamine ${ }^{3}$ or fentanyl ${ }^{4}$ are used for burn cases as an adjunct to general anaesthesia and in congenital heart disease to smooth the subsequent induction. By criteria of intragastric volume and $\mathrm{pH}$, the paediatric patient is at increased risk of aspiration. Risk factors can be decreased by use of $\mathrm{H}_{2}$ antagonists such as cimetidine. ${ }^{5}$ The patient with obesity or gastroesophageal reflux should be safer at induction if a blocker has been given as a premedicant.

As the practice of paediatric anaesthesia became more precise and expert, the advent of elective surgery for the "well" child began. The paediatric surgical patient of today is very different from the patient of an earlier period. Premedication may no longer be needed to ensure safety but it has an important role for many children in altering the stress associated with the operative and anaesthetic experience. Our patients are now most likely to be healthy, ambulatory, vocal and presenting for relatively minor surgery. The anxiety of such patients and their families may significantly affect their course in hospital. Children exhibit behaviourial and neurohumoral markers which indicate that the perioperative period is stressful. ${ }^{6}$ The widespread use of preoperative teaching and improved access of parents to the child have improved the surgical experience of many children but we are all too aware of the child who is still obviously distressed. There is a persistent need for pharmacological supplementation to prepare the frightened or unpredictable child. In addressing this need, we have avoided the invasive routes of $i m$ or $i v$ injection and have investigated less threatening methods - rectal, nasal, oral and transdermal. A wide range of sedatives and analgesics has been investigated in an attempt to make the process of separation and induction less anxious for the child and family.

Preoperative stress arises from pain or fear. Pain occurs inherently with some medical conditions and is caused by many of the investigations which are required preoperatively. Pain can be effectively relieved in the child with a variety of agents but the problem of how to deliver 
analgesics effectively and painlessly has beeen difficult to overcome. Nasal or oral fentanyl $l^{4}$ and ketamine ${ }^{3}$ are acceptable methods of inducing anaesthesia in a child and have the added advantage of providing some analgesia.

Anxiety and fear in the paediatric patient and family are often underestimated. The fears of the parents may be very different from those of their child but are manageable when the child is not anxious. Sedative agents can be very effective in minimizing distress. Rectal methohexitone and oral chloral hydrate are effective but have their limitations. Oral or nasal midazolam ${ }^{7,8}$ holds great promise as an agent that facilitates separation and induction. It has the added advantage that it may produce considerable amnesia for the preoperative period. This is a valuable effect for children who are very anxious or who may return to the OR often.

Pre-induction agents, especially analgesics and sedatives, may be unpredictable when administered to children. Mask induction with halothane usually produces a reliable state of anaesthesia after a predictable length of time. Premedicants given intramuscularly or absorbed through a mucosal surface can produce widely variable results in a seemingly similar population. Some children are relatively unaffected while others are obtunded. This may reflect our incomplete understanding of the absorption, volume of distribution and end organ effect of drugs we are beginning to give in unusual ways. The most important issues concern the respiratory and neurological compromise which occasionally occur. This concern has led to a gradual shift in premedication site from the ward to preinduction sedation under observation in an area close to equipment and personnel appropriate to care for the obtunded patient.

Premedication of the paediatric patient has altered dramatically since our earliest exposure to this population. It is now rarely required for the safe conduct of an anaesthetic. We have slowly begun to realize that while extensive preoperative psychological preparation is useful, it is not always enough to ensure a smooth induction. We are left with a large number of children who require pharmacological assistance and an increasing array of agents which enhance our handling of these patients. The expanding literature on premedication represents a real advance in anaesthesia practice. When agents and patients are properly selected premedication of the child is possible, effective and appropriate.

\section{Prémédication pédiatrique}

Dans la présente édition du Journal, McMillan et coll. ${ }^{1}$ décrivent l'utilisation du midazolam en prémédication chez les enfants. Ils démontrent que le midazolam per os est sécuritaire et efficace chez les enfants âgés de un à six ans devant subir une chirurgie ambulatoire. L'étude est bien faite et son message est réconfortant. Les enfants peuvent être sédationnés facilement et de façon sécuritaire en période pré-opératoire. Cet article vient s'ajouter aux nombreux autres portant sur la préparation de l'enfant avant la chirurgie. L'anesthésiste en pratique dispose maintenant d'un nombre croissant d'agents et de voies d'administration bien décrites dans la littérature et devrait pouvoir déterminer de façon appropriée un traitement chez l'enfant.

La prémédication chez l'enfant a suivi des modes: sédation lourde dans les premières années, aucune prémédication dans les années ' 70 et ' 80 et maintenant un retour à une sédation pré-induction. Est-ce que le nombre croissant d'études sur la sédation pédiatrique représente réellement une amélioration dans le traitement des patients? Ou est-ce tout simplement un exercice pharmacologique afin de déterminer quelles drogues sont absorbées par différentes muqueuses? Les indications pour les prémédications ont certe changé pour plusieurs de nos patients, mais elles existent toujours. Historiquement, l'administration d'une prémédication aux enfants était nécessaire pour l'administration sécuritaire d'une anesthésie générale. Dans une publication considérée comme un classique, Waters ${ }^{2}$ suggérait fortement de prémédiquer les enfants comme les adultes, c'est-à-dire avec morphine et scopolamine. En ces temps où le relâchement musculaire provenait surtout des anesthésiques volatils, la morphine était utilisée pour diminuer la concentration des agents d'inhalation requis et pour produire une stabilité cardiovasculaire améliorée. La scopolamine était très importante comme agent asséchant parce que l'éther provoquait des sécrétions copieuses. Contrairement à aujourd'hui, l'enfant de la pratique de Waters était plus souvent malade et les voies respiratoires n'étaient, en général, pas protégées. Suite au développement des relaxants musculaires et nouveaux agents d'inhalation, la nécessité absolue de ces prémédications est graduellement disparue chez le patient pédiatrique.

Encore de nos jours, il existe des indications de prémédication afin d'améliorer la sécurité de l'anesthésie générale. L'atropine est administrée dans le but d'assécher 
les sécrétions dans les cas d'intubation difficile et afin de maintenir une fréquence cardiaque adéquate pendant l'induction de l'anesthésie chez le nourrisson. La kétamine $^{3}$ ou le fentanyl ${ }^{4}$ sont utilisés dans les cas de brûlures comme adjuvants à l'anesthésie générale dans les cas de malformation cardiaque congénitale pour adoucir l'induction. Si on se base sur les critères de volume et de $\mathrm{pH}$ intragastriques, le patient pédiatrique est dans un groupe à risque élevé d'aspiration. Les facteurs de risque peuvent être diminués par l'utilisation des antagonistes $\mathrm{H}_{2}$ tel que la cimétidine. ${ }^{5}$ Le patient obèse ou présentant un reflux gastro-oesophagien aura une induction plus sécuritaire si un bloqueur a été administré en prémédication.

Suite au développement d'une anesthésie pédiatrique plus préçise et experte, nous avons assisté au développement de la chirurgie élective pour les enfants « en bonne sant ". De nos jours, le patient pédiatrique en chirurgie est très différent d'autrefois. Bien que la prémédication peut ne plus être nécessaire pour la sécurité de l'anesthésie, elle joue un rôle important chez plusieurs enfants afin de diminuer le stress associé à la chirurgie et à l'anesthésie. Nos patients sont plus sujets à être en santé, ambulants, «bruillants» et se présentant pour des chirurgies relativement mineures. L'anxiété de ces patients et de leurs familles peut affecter de façon significative leur séjour à l'hôpital. Certains traits de comportement et neurohumoraux des enfants indiquent que la période périopératoire est anxiogène. ${ }^{6}$ L'emploi généralisé de l'enseignement périopératoire et l'accès plus facile des parents à leurs enfants ont amélioré l'expérience chirurgicale de plusieurs enfants, même si nous sommes tous conscients de cet enfant qui est encore totalement désemparé. Il existe toujours un besoin de supplément pharmacologique afin de préparer l'enfant appeuré ou imprévisible. En se penchant sur ce besoin bien précis, nous avons évité les routes invasives telles que les injections $i m$ ou iv et avons étudié les méthodes moins menaçantes (rectale, nasale, orale ou transdermique). Plusieurs composés sédatifs et analgésiques ont été investigués afin de rendre ce processus de séparation et d'induction moins anxiogène pour l'enfant et la famille.

Le stress pré-opératoire est dû à la douleur ou à la peur. La douleur est causée soit par la condition médicale ellemême ou par les nombreuses investigations nécessaires dans la période pré-opératoire. La douleur peut être soulagée efficacement chez l'enfant à l'aide d'une variété d'agents, mais il est difficile de trouver une voie d'administration efficace et sans douleur pour les analgésiques. L'administration du fentany $1^{4}$ et de la kétamine $^{3}$ par voies nasale ou orale constitue des méthodes acceptables pour l'induction de l'anesthésie chez l'enfant, en plus d'offrir l'avantage d'un certain degré d'analgésie.
L'anxiété et la peur chez les patients pédiatriques et leurs familles sont souvent sous-estimées. Les peurs des parents peuvent être très différentes de celles de leurs enfants, mais sont contrôlables lorsque l'enfant n'est pas anxieux. Les agents sédatifs peuvent être très efficaces afin de diminuer cette détresse. Le méthohexital par voie rectale ou l'hydrate de chloral par voie orale sont efficaces mais ont leurs limitations. Le midazolam par voie orale ou nasale ${ }^{7,8}$ semble être un agent prometteur pour faciliter la séparation et l'induction. De plus, il a l'avantage de produire une amnésie considérable pendant la période préopératoire. Ceci est d'autant plus apprécié chez les enfants très anxieux et qui pourraient visiter souvent la salle d'opération.

Les agents administrés en pré-induction, surtout les analgésiques et sédatifs, peuvent être imprévisibles lorsqu'administrés aux enfants. L'induction au masque avec l'halothane produit en général un état d'anesthésie fiable dans un délai prévisible. Des agents donnés en prémédication par voie intramusculaire ou absorbés par la muqueuse peuvent produire des effets très variables chez une population à première vue semblable. Certains patients semblent relativement peu affectés tandis que d'autres sont assommés. Ceci est peut être secondaire à notre compréhension incomplète de l'absorption, du volume de distribution et des effets sur les organes effecteurs de médicaments que nous commençons à peine à administrer par des voies nouvelles. Les inquiétudes les plus fréquentes se rapportent aux effets négatifs rencontrés quelques fois sur les systèmes respiratoires et neurologiques. Pour prévenir ceci, la prémédication est de plus en plus administrée non plus à l'étage mais sous observation dans un endroit près de l'équipement et du personnel nécessaire pour les soins aux patients somnolents.

La prémédication du patient pédiatrique a changé de façon dramatique depuis nos premières armes face à cette population. Il est maintenant rare qu'une prémédication soit nécessaire pour l'administration sécuritaire d'un anesthésique. Nous avons graduellement réalisé qu'une préparation psychologique pré-opératoire extensive, quoiqu'utile, n'est pas toujours suffisante pour assurer une induction en douceur. Nous avons donc un grand nombre d'enfants qui nécessitent une assistance pharmacologique, et un arsenal croissant d'agents afin de nous aider à prendre soin de ces patients. Une littérature de plus en plus abondante sur la prémédication représente une amélioration importante dans la pratique de l'anesthésie. Lorsque les agents et les patients sont choisis avec soin, la prémédication de l'enfant est possible efficace et appropriée. 


\section{References}

1 McMillan CO, Spahr-Schopfer IA, Sikich N, Hartley E, Lerman J. Premedication of children with oral midazolam. Can J Anaesth 1992.

2 Waters RM. Pain relief for children. Am J Surg 1938; 470-5.

3 Gutstein HB, Johnson KL, Heard MB, Gregory GA. Oral ketamine preanesthetic medication in children. Anesthesiology 1992; 76: 28-33.

4 Feld LH, Champeau MW, van Steennis CA, Scott JC. Preanesthetic medication in children: a comparison of oral transmucosal fentanyl citrate versus placebo. Anesthesiology 1989; 71: 374-7.

5 Goudsouzian N, Cote CJ, Liu LMP, Dedrick DF. The dose-response effects of oral cimetindine on gastric $\mathrm{pH}$ and volume in children. Anesthesiology 1981; 55: 533-6.

6 Visintainer MA, Wolfer JA. Psychological preparation for surgical pediatric patients: the effect on children's and parents' stress responses and adjustment. Pediatrics 1975; 56: $1987-202$.

7 Wilton NCT, Leigh J, Rosen DR, Pandit UA. Preanesthetic sedation of preschool children using intranasal midazolam. Anesthesiology 1988; 69: 972-5.

$8 \mathrm{Karl} \mathrm{HW,} \mathrm{Keifer} \mathrm{AT,} \mathrm{Rosenberger} \mathrm{JL,} \mathrm{Larach} \mathrm{MG,}$ Ruffle JM. Comparison of the safety and efficacy of intranasal midazolam or sufentanil for preinduction of anesthesia in pediatric patients. Anesthesiology 1992; 76: 209-15. 